\title{
Research progress on the protective effects of fucoidan against intestinal mucosal barrier dysfunction (Review)
}

\author{
XIA QIU ${ }^{1,2}$, FAHE WANG $^{1,2}$, YIMIN QIN ${ }^{1,2}$, ZHANYI SUN ${ }^{1,2}$ and PEILI SHEN ${ }^{1,2}$ \\ ${ }^{1}$ State Key Laboratory of Bioactive Seaweed Substances, Qingdao Bright Moon Seaweed Group Co., Ltd.; \\ ${ }^{2}$ Qingdao Bright Moon Seaweed Bio-Health Technology Group Co., Ltd., Qingdao, Shandong 266400, P.R. China
}

Received October 28, 2021; Accepted December 21, 2021

DOI: $10.3892 /$ ijfn.2021.24

\begin{abstract}
The intestinal tract is one of the main organs responsible for digestion and absorption, and it also functions as a congenital barrier to maintain the homeostasis of the internal environment of the body. Damage to the intestinal mucosal barrier is closely related to the occurrence and development of a variety of diseases. As a natural food product, fucoidan has a number of biological activities. In recent years, it has been found that it exerts certain protective effects on intestinal physical, chemical, immune and microbial barriers. In the present review article, the role and mechanisms of action of fucoidan in protecting the intestinal mucosal barrier from damage are summarized and discussed. It is hoped that the information presented herein, may shed light onto the functions of fucoidan and may aid its use as a protective strategy against intestinal mucosal barrier dysfunction.
\end{abstract}

\section{Contents}

1. Introduction

2. Intestinal physical barrier

3. Intestinal immune barrier

4. Intestinal microbial barrier

5. Intestinal chemical barrier

6. Conclusions

\section{Introduction}

The intestinal tract is not only a crucial organ for nutrient uptake and utilization, but also constitutes a congenital

Correspondence to: Dr Xia Qiu, State Key Laboratory of Bioactive Seaweed Substances, Qingdao Bright Moon Seaweed Group Co., Ltd., 1 Daxueyuan Road, Qingdao, Shandong 266400, P.R. China E-mail: qiuqd@126.com

Key words: fucoidan, intestinal mucosal barrier, bacteria, intestinal flora, epithelial cells barrier to prevent harmful substances, such as pathogenic bacteria and toxins from entering the body. The intestinal barrier includes the following structures: The physical barrier, which mainly includes epidermal cells; the chemical barrier, which is comprised of intestinal fluid, mucin and digestive enzymes; the immune barrier, which includes immune cells and immune factors; and the microbial barrier, which is comprised of endogenous bacteria (1). The intestinal barrier has two main functions: On the one hand, it can prevent foreign antigens, microorganisms and toxic and harmful substances from entering the human body; on the other hand, as a selective filter of the body, it can transfer nutrients, electrolytes and water from the gut to the metabolic cycle of the body (2). An abnormal intestinal barrier function is closely related to the occurrence and development of various diseases.

Fucoidan is a unique active sulfated polysaccharide which normally contains fucose and sulfate groups, as well as galactose, xylose, mannose and uronic acids. It has two types of backbone chains. One type (I) encompasses repeated $(1 \rightarrow 3)$-linked $\alpha$-l-fucopyranose residues, and the other type (II) encompasses alternating and repeated $(1 \rightarrow 3)$-linke $\alpha$-l-fucopyranose and $(1 \rightarrow 4)$-linked $\alpha$-l-fucopyranose residues, essentially sulfated at positions 2 and/or 3 and/or 4, mainly found in the cell wall matrix, intercellular space and the secreted mucus of Sargassum stenophyllum, Laminaria hyperborea and other brown algae (3). In 2012, researchers were able to identify the chemical structure of fucoidan using nuclear magnetic resonance spectroscopy, or tandem electrospray ionisation mass spectrometry (ESI-MS) techniques (4). As a foodborne natural product, fucoidan has a variety of biological activities, such as anti-inflammatory, anticoagulant, antithrombotic, antiviral, immunomodulatory, antioxidant and antitumour activities (5). Different sources, seasonal variations or the methods of extraction can affect the chemical structure and molecular weight of fucoidan; in turn, its structure and molecular weight affect its biological activity (6).

In recent years, it has been found that fucoidan can maintain intestinal health by repairing the intestinal mucosa, enhancing immune function, regulating the intestinal flora, etc. It has also been shown to exert protective effects on intestinal physical, chemical, immune and microbial barriers (7) (Fig. 1). 


\section{Intestinal physical barrier}

The intestinal physical barrier includes intestinal dynamic function, intestinal epithelial cells and intercellular connections. It represents the first line of defence against the external environment, which is of utmost significance to the structure and function of the intestinal barrier (1). The functions of fucoidan in the intestinal physical barrier are summarized below.

Enhancement of intestinal power. Intestinal peristalsis, formed by the undulating contraction of the intestine, is an important part of the intestinal physical barrier. Following initial digestion in the stomach, food enters the small intestine, where it is absorbed by peristalsis. In a double-blind, randomized clinical trial, Matayoshi et al (8) reported that the administration of fucoidan ( $1 \mathrm{~g} /$ day) for 4 weeks significantly improved the number of defecations, the daily number of defecations, the soft and hard faeces index, and the volume of faeces in patients with constipation. The effect was more apparent in patients had taken fucoidan for 8 weeks. As a result, fucoidan from Nemacystus decipiens (Mozuku) can promote intestinal peristalsis, improve constipation, regulate the intestinal mechanical barrier (8).

Improves the histological morphology of the small intestine. The length of villi and the crypt depth of the small intestine are used to measure the digestive and absorption function of the small intestine, and the ratio of these indicates the comprehensive function of the small intestine. It was previously found that the daily administration of fucoidan extracted from Acaudina molpadioides improved the histological morphology of the small intestine damaged by cyclophosphamide and increased the villus length of the small intestine (9). The beneficial effect was more significant in the group treated with a molecular weight of $50 \mathrm{kDa}$ of fucoidan. Fucoidan and its enzymatic hydrolysates, among which the $50 \mathrm{kDa}$ fragment, exerted optimal effects in alleviating the weight loss caused by intestinal mucosal injury, and upregulating the expression of antibacterial peptides and stem cell marker genes. The mechanism responsible for its protective effects against chemotherapy-induced intestinal mucosal injury was found to be related to the Toll-like receptor-mediated MyD88 signalling pathway. In addition, fucoidan and its enzymatic hydrolysates also improved the villus/crypt ratio (V/C ratio; the ratio of the length of the villi and crypt depth of the small intestine) to varying degrees (9). Another study demonstrated that fucoidan increased the expression of intestinal tight junction proteins in a rat model of breast cancer, improving the structure of the intestine (10). The morphological structure of the intestine was significantly improved, and the damage to the intestinal mucosal physical barrier was also alleviated (10).

Repair of intestinal epithelial cells. Researchers have found that the pathogenic mechanisms of intestinal physical barrier injury may be that the dysfunction of cellular signalling pathways destroys key genes of intestinal epithelial cell differentiation, and the increased the susceptibility of patients to inflammatory bowel disease may be related to a lack of Notch 1 signalling (11). Lin et al (12) observed the effects of fucoidan on lipopolysaccharides and constructed a cell model of inflammatory injury of human colon epithelial cells. Their results revealed that the cell survival rate of the fucoidan intervention group significantly increased, and was positively associated with the fucoidan medium concentration. Fucoidan also attenuated the decrease in intestinal cell proliferation rate induced by inflammatory injury and repaired damaged intestinal epithelial cells (12). Furthermore, the effect was concentration-dependent.

\section{Intestinal immune barrier}

The intestine is the largest immune organ in the body, and $25 \%$ of the intestinal mucosa is composed of lymphoid tissue, which plays an important role in the intestinal immune barrier against bacterial invasion. A summary of the effects of fucoidan on the intestinal immune barrier is presented below.

Activation of intestinal immune cells. Researchers have found that fucoidan can stimulate the proliferation, differentiation and maturity of immunoactive cells, regulates the Th1/Th2 ratio of the intestinal helper $\mathrm{T}$-cells, protects the intestinal immune barrier and reduce the body's inflammatory response, as evidenced by a series of scientific experiments (12). Shang et al (13) found that fucoidan reduced the antigen load of the body, relieved the inflammatory response of the host, increased the expression of immunoglobulin A ( $\operatorname{Ig} \mathrm{A})$ and reduced the level of serum lipopolysaccharide binding protein. O'Shea et al (14) established an experimental porcine model of colitis induced by dextran sodium sulfate (DSS), and conducted intragastric intervention with fucoidan to observe the effects of on the pathological changes of colitis. It was found that fucoidan increased the body weight, and improvised the proximal colon pathological score and diarrhoea score of the pigs, also reducing the abundance of IL-6 mRNA in the colon (14).

Regulation of the expression of cytokines. There is evidence to indicate that fucoidan regulates the expression of certain cytokines in the intestinal mucosa. Park et al (15) found that fucoidan enhanced the intestinal immune barrier and anti-inflammatory function by inhibiting the secretion of IL-1 $\beta$, IL-13, TNF- $\alpha$, IL-6, IFN- $\gamma$ and other inflammatory factors by macrophages. It has been reported that the oral administration of fucoidan decreases the synthesis of pro-inflammatory cytokines and exerts protective effects on intestinal barrier function in non-obese diabetic mice (16). Fucoidan was also shown to enhance intestinal acquired immunity by increasing the expression of the IL-6 and IL-10 cytokines (7). In addition, through in vitro and in vivo experiments, it has also been found that fucoidan can directly interact with Toll-like receptors of immune cells to produce mucin and enhance the immune response (17).

Promotes immune effector cells. Fucoidan can promote the activation of immune effector cells, regulate the intestinal immune barrier and improve the immune capacity of the body, thus playing an auxiliary therapeutic role in inhibiting intestinal tumours and alleviating the side-effects of chemotherapy. Usoltseva et al (5) found that fucoidan and its derivatives inhibited the growth of human colorectal adenocarcinoma epithelial 


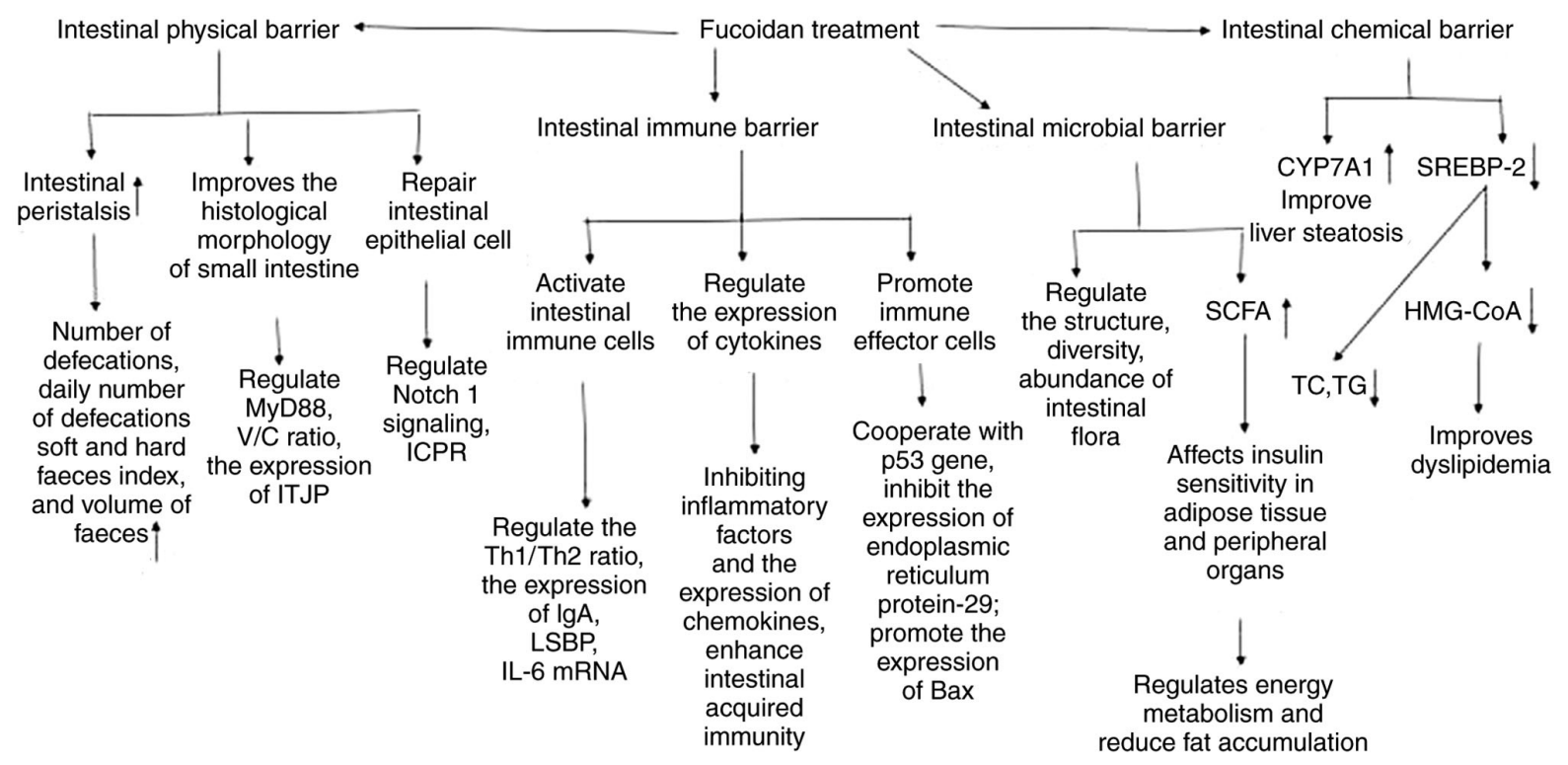

Figure 1. Schematic representation of the functions of fucoidan in the intestinal barrier. MyD88, myeloid differentiation primary response gene (88; Toll-like receptor-mediated signalling pathway); V/C ratio, villus/crypt ratio (the ratio of the length of the villi and crypt depth of the small intestine); ITJP, intestinal tight junction protein; ICPR, intestinal cell proliferation rate; Th1/Th2, T helper type 1/2; IgA, immunoglobulin A; LSBP, lipopolysaccharide binding protein; IL-6 mRNA, interleukin-6 messenger RNA; ERP-29, endoplasmic reticulum protein-29; Bax, apoptotic protein Bax; SCFA, short chain fatty acid; CYP7A1, cholesterol $7 \alpha$-hydroxylase; SREBP-2, sterol regulatory element binding protein 2; HMG-CoA, hydroxy methylglutaryl coenzyme A reductase; TC, total cholesterol; TG, triglyceride.

cells and human colon cancer cells in in vitro cell experiments. It was found that fucoidan can play an anticancer role independently of the p53 gene, and can also cooperate with the p53 gene to exert anticancer effects (5). Chen et al (18) found that fucoidan inhibited the expression of endoplasmic reticulum protein-29 in colon cancer cells, promoted the expression of the apoptotic protein, Bax and induced the apoptosis of colon cancer cells.

\section{Intestinal microbial barrier}

There are a large number of symbiotic bacteria attached to the mucosal layer of the intestinal surface in the human intestine, which stimulates the intestinal epithelial cells to secrete a variety of substances, forms a dynamic and stable microecosystem with the host. This is known as the intestinal microbial barrier, which plays an important role in maintaining human health. It is a necessary factor for the maintenance of human health, and also reflects the stable state of the internal environment of the body (1). The effects of fucoidan on the functions of the intestinal microbial barrier are briefly discussed below.

Reshaping the intestinal flora. Experimental studies have found that fucoidan can alter the structure, diversity and abundance of the intestinal flora, thus protecting intestinal microbial barrier function (10). Shi et al (9) found that the addition of fucoidan $(50 \mathrm{mg} / \mathrm{kg}$ body weight) to the daily diet altered the intestinal flora of normal mice, increased the diversity of the flora, and significantly increased the number and abundance of probiotics, such as Coprococcus, Rikenellaceae and Butyricicoccus in the intestinal tract of mice. It also reduce the relative number and abundance of harmful bacteria, such as Proteobacteria, Firmicutes and Corynebacterium, thus attenuating intestinal mucosal injury (9).
Adjuvant treatment of disease through intestinal microbiome mediation. A series of studies have suggested that fucoidan can be used as a microecological modulator in the adjuvant treatment of some diseases mediated by the intestinal flora (19). It has been found that fucoidan may be used as an intestinal microbiota regulator to promote health and to treat intestinal dysfunction, significantly reduce the host's antigen load and inflammatory response, and reduce the level of serum lipopolysaccharide binding protein (15). Liu et al (20) demonstrated that fucoidan extracted from Undaria pinnatifida significantly reduced the abundance of Staphylococcus in the intestinal tract, which was positively associated with dyslipidaemia, and also significantly increased the abundance of Prevotella, which was negatively associated with obesity. Chen et al (21) found that the supplementation of algae polysaccharide reduced the abundance and number of Corynebacterium, Brevibacterium and Aerococcus caused by a high-fat diet, and effectively restored the disruption of intestinal flora homeostasis induced by a high-fat diet in rats, and improve dyslipidaemia. It has also been found that the number of Akkermansia decreased significantly in pre-diabetic and diabetic patients, while the bacteria were significantly enriched following intervention with fucoidan in diabetic model mice; the decrease in the number Akkermansia was significantly improved, and metabolic disorders, such as insulin resistance were reversed (22). Another study using animal models proved that fucoidan derived from Laminaria Japonica significantly reduced weight gain, attenuated liver steatosis and alleviated inflammation (23). Further analysis of the mechanisms from the perspective of intestinal flora revealed that fucoidan enriched the beneficial bacteria, such as Alloprevotella, Akkermansia, Blautia and Bacteroidetes, and increased the production of short-chain fatty acids (SCFAs). SCFAs are ligands of G-protein-coupled receptors, which can affect insulin sensitivity in adipose tissue 
and peripheral organs, thereby regulating energy metabolism and reducing fat accumulation (23).

\section{Intestinal chemical barrier}

The intestinal chemical barrier mainly consists of the mucus layer, which alters the locus of gut microbes and prevents them from directly contacting with host intestinal tissue cells. In addition, certain substances produced in the intestinal tract, such as bile salt, mucopolysaccharides, lysozyme, glycoproteins, etc. also play a certain role in the chemical barrier. Defects in the chemical barrier can cause inflammatory injury to intestinal epithelial cells, and the anatomical site of damaged mucus layer is related to the position of intestinal injury in morphology (24). Chen et al (21) examined the mechanisms of action of fucoidan from Undaria pinnatifida to improve the lipid level of model animals with dyslipidaemia, and found that fucoidan increased the synthesis of the rate-rate-releasing enzyme, cytochrome P450 family 7 subfamily A member 1 (CYP7A1), by hepatic bile acid that affected lipid metabolism, and attenuate liver steatosis induced by a high-fat diet. It was also found that fucoidan reduced the total cholesterol and triglyceride levels of the liver by decreasing the expression of sterol regulatory element binding protein 2 (SREBP-2) related to cholesterol biosynthesis. SREBP-2 can selectively activate de novo cholesterol synthesis by inducing the gene transcription of HMG-CoA Reductase (HMGCR) and other enzymes of the cholesterol synthesis pathway. Therefore, fucoidan may improve dyslipidaemia by mediating HMGCR and SREBP-2 (21).

\section{Conclusions}

As a natural functional food, fucoidan exerts notable protective effects on the intestinal physical, chemical, immune and microbial barrier. Firstly, fucoidan can promote intestinal peristalsis, improve the morphological structure of the small intestine and repair intestinal epithelial cells. Secondly, fucoidan can activate intestinal immune cells, regulate the expression of cytokines and promote immune effector cells. Thirdly, it can promote the proliferation of intestinal beneficial flora, inhibit the implantation of harmful flora, regulate the structure and metabolic function of intestinal flora, and can thus prevent and treat certain diseases through intestinal flora mediation. Finally, fucoidan can reduce intestinal permeability, repair intestinal mucosal inflammation, regulate bile acid synthesis and affect lipid metabolism.

As fucoidan cannot be digested and degraded in the stomach and small intestine, it is biotransformed by bacteria in the large intestine to perform its biological function. Various fucoidans have different sources and extraction methods, and their molecular weight and fructose composition also differ; thus, their effects on the intestinal barrier also differ to a certain extent (25). In the future, further basic and clinical studies are warranted to verify the mechanisms of action of fucoidan in protecting the intestinal barrier, and explore its development and application as a microecological regulator.

Fucoidan has now become a commercial product that can be purchased by individuals. Fucoidan can be used in dietary supplements, energy drinks, beauty and skin care products and animal nutrition, as well as in other areas. It is considered to have immense value and potential for market application in the health industry (19).

\section{Acknowledgements}

Not applicable.

\section{Funding}

The present study was supported by the Major Scientific and Engineering Projects of Innovation in Shandong Province (grant no. 2019JZZY010818) and the 2020 Science and Technology Project of Qingdao West Coast New District (2020-3-1).

\section{Availability of data and materials}

Not applicable.

\section{Authors' contributions}

XQ was involved in the conceptualization of the study, in the of the writing original draft, and in the writing, reviewing and editing of the manuscript. FW was involved in the design of the study and in funding acquisition. YQ was involved in the conceptualization of the study and in funding acquisition. ZS was involved in the format conversion of the figure and article, and in the literature search. PS was involved in the literature search and collation. All authors have read and approved the final manuscript. Data authentication is not applicable.

\section{Ethics approval and consent to participate}

Not applicable.

\section{Patient consent for publication}

Not applicable.

\section{Competing interests}

The authors declare that they have no competing interests.

\section{References}

1. Ma X, Dai Z, Sun K, Zhang Y, Chen J, Yang Y, Tso P, Wu G and $\mathrm{Wu} Z$ : Intestinal epithelial cell endoplasmic reticulum stress and inflammatory bowel disease pathogenesis: An update review. Front Immunol 8: 1271-1273, 2017.

2. Blikslager AT, Moeser AJ, Gookin JL, Jones SL and Odle J: Restoration of barrier function in injured intestinal mucosa. Physiol Rev 87: 545-564, 2007.

3. Li B, Lu F, Wei X and Zhao R: Fucoidan: Structure and bioactivity. Molecules 13: 1671-1695, 2008.

4. Anastyuk SD, Imbs TI, Shevchenko NM, Dmitrenok PS and Zvyagintseva TN: ESIMS analysis of fucoidan preparations from Costaria costata, extracted from alga at different life-stages. Carbohydr Polym 90: 993-1002, 2012.

5. Usoltseva RV, Anastyuk SD, Ishina IA, Isakov VV, Zvyagintseva TN, Thinh PD, Zadorozhny PA, Dmitrenok PS and Ermakova SP: Structural characteristics and anticancer activity in vitro of fucoidan from brown alga Padina boryana. Carbohydr Polym 184: 260-268, 2018. 
6. Wu L, Sun J, Su X, Yu Q, Yu Q and Zhang P: A review about the development of fucoidan in antitumor activity: Progress and challenges. Carbohydr Polym 154: 96-111, 2016.

7. Zuo T, Li X, Chang Y, Duan G, Yu L, Zheng R, Xue C and Tang Q: Dietary fucoidan of Acaudina molpadioides and its enzymatically degraded fragments could prevent intestinal mucositis induced by chemotherapy in mice. Food Funct 6: 415-422, 2015.

8. Matayoshi M, Teruya J, Yasumoto-Hirose M, Teruya R, Miura N and Takeda R: Improvement of defecation in healthy individuals with infrequent bowel movements through the ingestion of dried Mozuku powder: A randomized, double-blind, parallel-group study. Funct Foods Health Dis 7: 735-742, 2017.

9. Shi H, Chang Y, Gao Y, Wang X, Chen X, Wang Y, Xue C and Tang Q: Dietary fucoidan of Acaudina molpadioides alters gut microbiota and mitigates intestinal mucosal injury induced by cyclophosphamide. Food Funct 8: 3383-3393, 2017.

10. Xue M, Ji X, Liang H, Liu Y, Wang B, Sun L and Li W: The effect of fucoidan on intestinal flora and intestinal barrier function in rats with breast cancer. Food Funct 9: 1214-1223, 2018.

11. Wang Y, Huang D, Chen KY, Cui M, Wang W, Huang X, Awadellah A, Li Q, Friedman A, Xin WW, et al: Fucosylation deficiency in mice leads to colitis and adenocarcinoma. Gastroenterology 152: 193-205, 2017.

12. Liu HJ, Li HT, Lin Y, Lu DL, Yue Y, Xiong J, Li CQ, Xu XY and Feng YG: Identification of 2 potential core genes for influence of gut probiotics on formation of intracranial aneurysms by bioinformatics analysis. Med Sci Monit 26: e920754, 2020.

13. Shang Q, Shan X, Cai C, Hao J, Li G and Yu G: Dietary fucoidan modulates the gut microbiota in mice by increasing the abundance of Lactobacillus and Ruminococcaceae. Food Funct 7 : 3224-3232, 2016.

14. O'Shea CJ, O'Doherty JV, Callanan JJ, Doyle D, Thornton K and Sweeney T: The effect of algal polysaccharides laminarin and fucoidan on colonic pathology, cytokine gene expression and Enterobacteriaceae in a dextran sodium Sulfate-challenged porcine model. J Nutr Sci 5: e15, 2016.

15. Park J, Cha JD, Choi KM, Lee KY, Han KM and Jang YS: Fucoidan inhibits LPS-induced inflammation in vitro and during the acute response in vivo. Int Immunopharmacol 43: 91-98, 2017

16. Sun T, Liang H, Xue ML, Liu Y, Gong A, Jiang Y, Qin Y, Yang J and Meng D: Protective effect and mechanism of fucoidan on intestinal mucosal barrier function in NOD mice. Food Agr Immunol 31: 922-936, 2020.
17. Okolie CL, Rajendran SRCK, Udenigwe CC, Aryee ANA and Maso B: Prospects of brown seaweed polysaccharides (BSP) as prebiotics and potential immunomodulators. J Food Biochemistry 7: e12392, 2017.

18. Chen SH, Zhao Y, Zhang Y and Zhang D: Fucoidan induces cancer cell apoptosis by modulating the endoplasmic reticulum stress cascades. PLoS One 9: e108157, 2014.

19. Kan J, Cheng J, Xu L, Hood M, Zhong D, Cheng M, Liu Y, Chen $\mathrm{L}$ and $\mathrm{Du} \mathrm{J}$ : The combination of wheat peptides and fucoidan protects against chronic superficial gastritis and alters gut microbiota: A double-blinded, placebo-controlled study. Eur J Nutr 59: 1655-1666, 2020.

20. Liu M, Ma L, Chen Q, Zhang P, Chen C, Jia L and Li H: Fucoidan alleviates dyslipidemia and modulates gut microbiota in high-fat diet-induced mice. J Funct Foods 48: 220-227, 2018.

21. Chen Q, Liu M, Zhang P, Fan S, Huang J, Yu S, Zhang C and Li H: Fucoidan and galactooligosaccharides ameliorate high-fat diet-induced dyslipidemia in rats by modulating the gut microbiota and bile acid metabolism. Nutrition 65: 50-59, 2019.

22. Blandino G, Inturri R, Lazzara F, Di Rosa M and Malaguarnera L: Impact of gut microbiota on diabetes mellitus. Diabetes Metab 42: 303-315, 2016.

23. Shang Q, Song G, Zhang M, Shi J, Xu C, Hao J, Li G and Yu G: Dietary fucoidan improves metabolic syndrome in association with increased Akkermansia population in the gut microbiota of high-fat diet-fed mice. J Funct Foods 28: 138-146, 2017.

24. Zhang J, Ankawi G, Sun J, Digvijay K, Yin Y, Rosner MH and Ronco C: Gut-kidney crosstalk in septic acute kidney injury. Crit Care 22: 117-120, 2018.

25. Kadena K, Tomori M, Iha M and Nagamine T: Absorption study of Mozuku fucoidan in Japanese volunteers. Mar Drugs 16: 254-258, 2018.

This work is licensed under a Creative Commons Attribution-NonCommercial-NoDerivatives 4.0 International (CC BY-NC-ND 4.0) License. 